\title{
Why Do Liquids Mix? The Mixing of Protic Ionic Liquids Sharing the Same Cation is Apparently Driven by Enthalpy, not Entropy
}

\author{
Benjamin Golub, ${ }^{1}$ Daniel Ondo, ${ }^{2}$ Ralf Ludwig, ${ }^{1,3}$ and Dietmar Paschek ${ }^{1, *}$ \\ ${ }^{1}$ Physikalische und Theoretische Chemie, Institut für Chemie, \\ Universität Rostock, Albert-Einstein-Straße 27, D-18059 Rostock, Germany \\ ${ }^{2}$ Department of Physical Chemistry, University of Chemistry and Technology, Technická 5., 16628 Prague 6, Czech Republic \\ ${ }^{3}$ Leibniz-Institut für Katalyse, Albert-Einstein-Straße 29a, D-18059 Rostock, Germany
}

(Dated: 2022/03/01 at 18:23:41)

\begin{abstract}
We study hydrogen bond (HB) redistribution in mixtures of two protic ionic liquids (PILs) sharing the same cation: triethylammonium-methanesulfonate ([TEA][OMs]) and triethylammonium-trifluoromethanesulfonate ([TEA][OTf]). The mixture is exhibiting large negative energies of mixing. Based on results obtained from atomic detail molecular dynamics (MD) simulations, we derive a lattice model, discriminating between HB and nonspecific intermolecular interactions. We demonstrate that due to the ordered structure of the PILs, only the $\mathrm{HB}$ interactions contribute to the mixing energy. This allows to us to connect the equilibrium of HBs to each of the two anion species with the corresponding excess energies and entropies. The entropy associated with HB redistribution is shown to be negative, and even overcompensating the positive entropy associated with a statistical distribution of the ions in the mixture. This is strongly suggesting that the mixing process is driven by enthalpy, not entropy.
\end{abstract}

Protic ionic liquids (PILs) [1] have attracted considerable interest since they possess qualities such as biocompatibility [2] and low toxicity.[3] Mixtures of ionic liquids (ILs) have gained increasing attention in recent years.[4-19] Mixtures of ILs are often characterized by their general mixing behavior, analysing to what degree they are deviating from Raoult's law (i.e. ideal mixing behavior).[20] As a rule of thumb, ILs often mix well.[20] However, whether we find a nonideal mixing behavior is, of course, depending on the interaction forces between the ions. Combinations of strong dispersion forces [21], but also hydrogen bonding [22, 23], can lead to a significant nonideal mixing. A direct way to investigate the formation or change of interactions due to mixing is to determine the enthalpy or energy of mixing.[20-29] If the enthalpy of mixing is close to zero, the mixing is, of course, nearly ideal. Positive or negative enthalpies of mixing, however, imply the "making or breaking" [30] of interactions and are therefore often related with changes of the structure of the liquid.[31] Extensive reviews of mixtures of ionic liquids are available in refs [20, 26, 30].

In this study we would like to focus on a specific mixture of PILs, which has the potential to disentangle the contribution from hydrogen bonding and other nonspecific forms of intermolecular interactions: the mixture of two rather common protic ionic liquids sharing the same cation. The two PILs are triethylammonium-methanesulfonate ([TEA][OMs]) and triethylammonium-trifluoromethanesulfonate ([TEA][OTf]). In the PIL, a [TEA] cation acts as a hydrogen bond (HB) donor, being able to donate a single HB. Both, the [OMs] and the [OTf] anions can act as HB acceptors, which can accept multiple $\mathrm{HBs}$ via their respective $\mathrm{SO}_{3}$-groups. In previous publications $[11,12,32]$ we could demonstrate that a mixture of those PILs experiences a redistribution of HBs between the cation and the anions in the mixture apparently due to stronger HBs between [TEA] and [OMs] ions [12, 33, 34]. This is leading to nonideal mixing behavior, almost com- pletely stripping the [OTf] anion of HB partners in the [OMs]rich region.[11] Note that the local environment of each of the anions is formed by a solvation shell composed predominantly of cations. Hence the environment of each anion is very similar in the mixture compared to the respective pure PILs, such that the nonideal mixing behavior is most likely only due to HB redistribution effects.

To study the pure PILs and mixtures, we performed isobaric isothermal (NPT) molecular dynamics (MD) simulations investigating eleven compositions between $x_{\mathrm{OMs}}=0.0$ and $x_{\mathrm{OMs}}=1.0$ for five temperatures between $T=320 \mathrm{~K}$ and $T=400 \mathrm{~K}$ at a pressure of $1 \mathrm{bar}$. All studied systems were composed of 500 ion pairs. More details about the performed MD simulations are provided in the SI.

In a previous contribution we have shown that MD simulations of pure [TEA][OMs] and [TEA][OTf] as well as their mixtures exhibit a well defined charge-induced intermolecular order.[11] Here each ion was found to be surrounded by a solvation shell predominantly consisting of counter ions. The average coordination number, defined by a center of mass distance of $0.8 \mathrm{~nm}$ between ions of opposite charge (corresponding to the location of the first minimum of anion-cation pair correlation functions) was found to vary only slightly between 6.8 and 6.9 as a function of the mixture composition. The corresponding distribution functions (see Figure 6 of ref [11]), are characterized by a maximum at seven neighbors with a half width of about 2.5 neighbors. A representative snapshot of the solvation of a [TEA] cation in [TEA][OMs]/[TEA][OTf] mixture is shown in Figure 1a.

HBs are characterized here by means of geometric criteria. Based on the analysis discussed in ref [11], we use a distance criterion of $r_{\mathrm{HN} . . \mathrm{O}} \leq 0.24 \mathrm{~nm}$ to define a $\mathrm{HB}$ for both, the [OMs] and the [OTf] ions. We obtain the standard enthalpy associated with switching a HB between the two anion species from a van 't Hoff plot of the logarithm of the equilibrium constant $K=y_{\mathrm{OMs}} / y_{\mathrm{OTf}}$ versus the inverse temperature shown in 

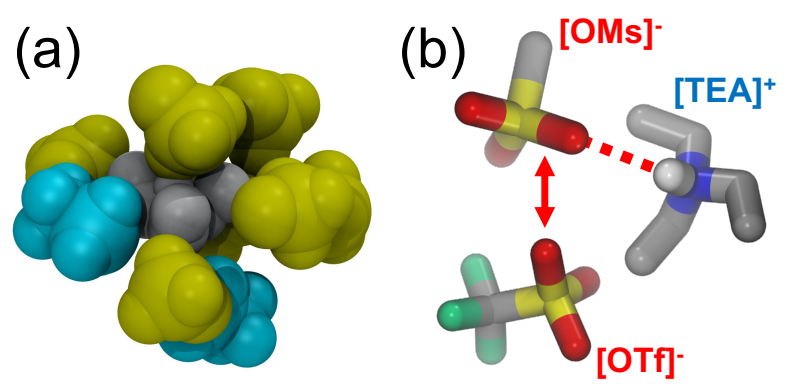

FIG. 1. a) Snapshot of the solvation of a central [TEA] cation, shown in gray, in a [TEA][OMs]/[TEA][OTf] mixture engaged in a $\mathrm{HB}$ to an adjacent [OMs] anion. The [OMs] anions are shown in yellow and the [OTf] anions are depicted in cyan. b) Illustrated equilibrium of the HBs donated by a [TEA] cation and accepted by the $\mathrm{SO}_{3}$ groups of the [OMs] and [OTf] anions.

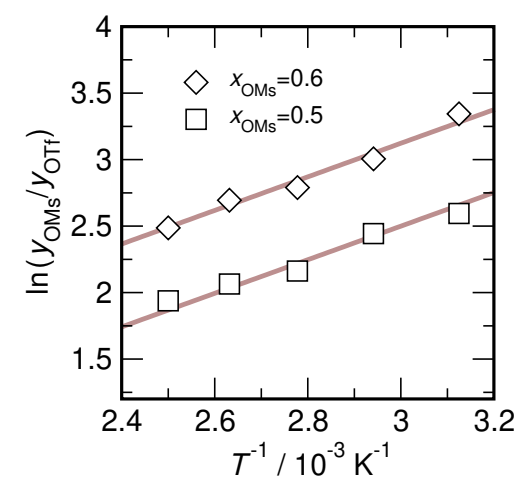

FIG. 2. Van 't Hoff plot of the logarithm of the equilibrium constant $K=y_{\mathrm{OMs}} / y_{\mathrm{OTf}}$ vs. the inverse temperature for compositions with $x_{\mathrm{OMs}}=0.5$, and $x_{\mathrm{OMs}}=0.6$. The slope of the solid lines correspond to a $\Delta H^{\circ}$ of $-10.5 \mathrm{~kJ} \mathrm{~mol}^{-1}$.

Figure 2. Here $y_{\mathrm{OMs}}$ and $y_{\mathrm{OTf}}$ represent the fraction of HBs donated by the [TEA] cation to either of the two anion species. Depicted are data for compositions of $x_{\mathrm{OMs}}=\{0.5,0.6\}$, indicating a value of about $\Delta H^{\circ}=-10.5 \mathrm{~kJ} \mathrm{~mol}^{-1}$ for the enthalpy associated with breaking a [TEA][OTf] HB and forming a new [TEA][OMs] HB.

From the energies recorded during the MD simulations we can directly determine the energies of mixing according to

$$
\begin{aligned}
\Delta U_{\text {mix }}= & U\left(x_{\mathrm{OMs}}\right)-x_{\mathrm{OMs}} \cdot U_{[\mathrm{TEA}][\mathrm{OMs}]} \\
& -\left(1-x_{\mathrm{OMs}}\right) \cdot U_{[\mathrm{TEA}][\mathrm{OTf}]},
\end{aligned}
$$

where $U\left(x_{\mathrm{OMs}}\right)$ represents the average total energy of the mixture [TEA][OTf]/[TEA][OMs] with a given composition $x_{\mathrm{OMs}}$, while $U_{\text {[TEA][OTf] }}$ and $U_{\text {[TEA][OMs] }}$ are the average total energies of the pure PILs. The MD simulations indicate a strong nonideal mixing behavior. The computed negative mixing energies of the equimolar mixture are found to be $(-4.02 \pm 0.18) \mathrm{kJ} \mathrm{mol}^{-1}$ at $400 \mathrm{~K}$ and $(-4.50 \pm$ $0.21) \mathrm{kJ} \mathrm{mol}^{-1}$ at $320 \mathrm{~K}$ as shown in Table S12 in the SI. In addition, we observe that the energy minima are shifted to a $x_{\mathrm{OMs}}$ value of about $x_{\mathrm{OMs}}=0.4$, which is almost temperature independent.

The regular local structure of the PILs is characterized by a well defined anion-cation order as discussed in ref [11]. This has inspired us to describe the mixture in terms of a lattice model based solely on local nearest neighbor interactions. In the model we discriminate between HBs and nonspecific interactions (i.e. van der Waals and non-localized polar interactions). Here each of the ions in the PILs is placed on a regular lattice with a coordination number $c$. As illustrated in Figure 3 the anions and cations are arranged in an alternating fashion on the lattice. Hence there exist $\left(N_{\mathrm{TEA}} \cdot c\right)$ nonspecific nearest neighbor interactions and ( $\left.N_{\mathrm{TEA}}\right)$ HBs. The total energy of the pure PILs follows as

$$
\begin{aligned}
U_{[\mathrm{TEA}][\mathrm{OMs}]}= & N_{\mathrm{TEA}} \cdot c \cdot \epsilon_{\mathrm{TEA}-\mathrm{OMs}}+ \\
& N_{\mathrm{TEA}} \cdot \epsilon_{\mathrm{HB}: \mathrm{TEA}-\mathrm{OMs}}
\end{aligned}
$$

and

$$
\begin{aligned}
U_{[\mathrm{TEA}][\mathrm{OTf}]}= & N_{\mathrm{TEA}} \cdot c \cdot \epsilon_{\mathrm{TEA}-\mathrm{OTf}}+ \\
& N_{\mathrm{TEA}} \cdot \epsilon_{\mathrm{HB}: \mathrm{TEA}-\mathrm{OTf}},
\end{aligned}
$$

where $N_{\text {TEA }}$ is the number of [TEA] cations, $c$ is the coordination number, and $\epsilon_{\text {TEA-OMs }}, \epsilon_{\text {TEA-OTf }}, \epsilon_{\mathrm{HB} \text { :TEA-OMs }}$, and $\epsilon_{\mathrm{HB} \text { :TEA-OTf }}$ are the parameters characterising the respective nonspecific and HB interactions of the cation with either of the two anions. Assuming that in a mixture the sites of the anion sublattice are statistically occupied by one of the two anion species in accordance with the mixture composition, the energy of the mixture with a composition $x_{\mathrm{OMs}}$ is given by

$$
\begin{aligned}
U\left(x_{\mathrm{OMs}}\right)= & N_{\mathrm{TEA}} \cdot c \cdot x_{\mathrm{OMs}} \cdot \epsilon_{\mathrm{TEA}-\mathrm{OMs}}+ \\
& N_{\mathrm{TEA}} \cdot y_{\mathrm{OMs}} \cdot \epsilon_{\mathrm{HB}: \mathrm{TEA}-\mathrm{OMs}}+ \\
& N_{\mathrm{TEA}} \cdot c \cdot\left(1-x_{\mathrm{OMs}}\right) \cdot \epsilon_{\mathrm{TEA}-\mathrm{OTf}}+ \\
& N_{\mathrm{TEA}} \cdot\left(1-y_{\mathrm{OMs}}\right) \cdot \epsilon_{\mathrm{HB}: \mathrm{TEA}-\mathrm{OTf}},
\end{aligned}
$$

where $y_{\mathrm{OMs}}$ represents the fraction of cations forming a $\mathrm{HB}$ to the neighboring [OMs] anions. Note that in the present case the condition $y_{\mathrm{OMs}}+y_{\mathrm{OTf}}=1$ is well fulfilled. From Equations 1, 2, 3, and 4 follows directly that

$$
\Delta U_{\mathrm{mix}}=N_{\mathrm{TEA}} \cdot\left(y_{\mathrm{OMs}}-x_{\mathrm{OMs}}\right) \cdot \Delta \epsilon_{\mathrm{HB}}
$$

with

$$
\Delta \epsilon_{\mathrm{HB}}=\epsilon_{\mathrm{HB}: \mathrm{TEA}-\mathrm{OMs}}-\epsilon_{\mathrm{HB}: \mathrm{TEA}-\mathrm{OTf}} .
$$

Note that $\Delta \epsilon_{\mathrm{HB}}<0$ for the case of $\epsilon_{\mathrm{HB}: \mathrm{TEA}-\mathrm{OMs}}<$ $\epsilon_{\mathrm{HB}: T E A-O T f}$ is leading to negative mixing energies if the condition $y_{\mathrm{OMs}}>x_{\mathrm{OMs}}$ is fulfilled. It is evident from Equation 5 that the difference of the hydrogen bonding strengths of HBs donated by the [TEA] cation to each of the two anion species can thus be obtained from the knowledge of $\Delta_{\text {mix }} U$, $y_{\mathrm{OMs}}$ and $x_{\mathrm{OMs}}$.

The lattice model outlined in Figure 3 also allows us to derive the entropic contributions due to HB redistribution. First, let us assume that the anions in the mixture occupy the anion positions on the anion sublattice randomly according to 
(a)

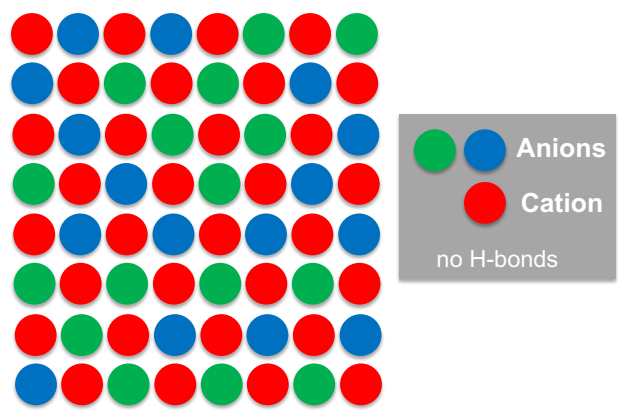

(b)

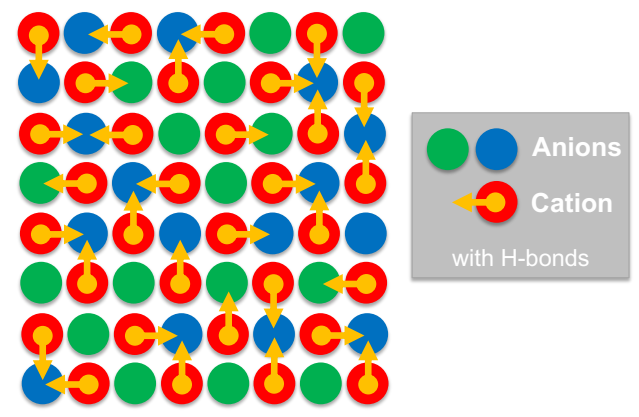

FIG. 3. 2D lattice representation (with coordination number $c=4$ ) of an equimolar mixture of [TEA][OMs] and [TEA][OTf]. The color coding is: red $=[\mathrm{TEA}]^{+}$, blue $=[\mathrm{OMs}]^{-}$, and green $=[\mathrm{OTf}]^{-}$. a) Lattice sites are occupied by cations and anions in an alternate fashion, leading to a number of nonspecific cation-anion contacts of $N_{[\mathrm{TEA}]} \cdot c$. The anions are randomly distributed on the lattice-sites of the anion-sublattice according to their mixture composition. b) The arrows indicate HBs between the cations and one of the adjacent anions, therefore representing one additional HB interaction per cation. Note that the majority of $\mathrm{HBs}$ is found between [TEA] cations and [OMs] anions.
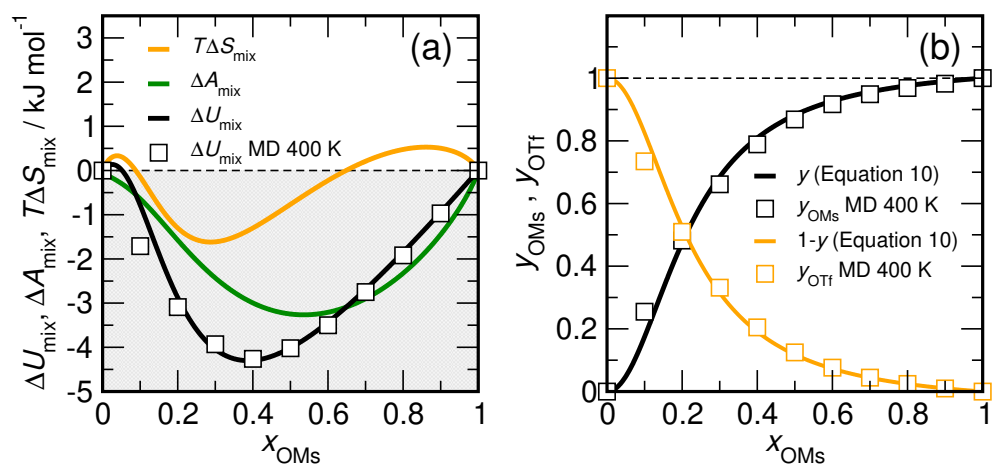

FIG. 4. Theoretical predictions of the thermodynamic mixing properties for mixtures at $T=400 \mathrm{~K}$ using $\Delta \epsilon_{\mathrm{HB}}=-10.5 \mathrm{~kJ}$ mol ${ }^{-1}$ and $\alpha=2.05$. a) Open squares indicate the energies of mixing as function of $x_{\mathrm{OMs}}$ obtained from MD simulations. The heavy solid lines represent the $\Delta A_{\text {mix }}, \Delta U_{\text {mix }}$ and $T \Delta S_{\text {mix }}$ according to Equations 13, 11, and 14, respectively. b) Shown are the fraction $y_{\mathrm{OMs}}$ and $y_{\mathrm{OTf}}$ of [TEA] cations involved in a HB with [OMs] and [OTf] anions. Open circles represent data obtained from MD simulations. The heavy solid line represents data according to Equation 10.

their mixture composition. Since none of the nonbonded interactions change during the mixing process, the corresponding energy of mixing is $\Delta U_{\text {mix-id. }}=0$, and

$$
\Delta S_{\text {mix-id. }}=-N k_{\mathrm{B}}[x \ln (x)+(1-x) \ln (1-x)]
$$

is the associated ideal mixing entropy contribution due to the statistical distribution of the anions on the anion sublattice, with $x=x_{\mathrm{OMs}}$ and $k_{\mathrm{B}}$ being the Boltzmann constant and $N$ representing the total number of anions in the mixture. Hence

$$
\Delta A_{\text {mix-id. }}=N k_{\mathrm{B}} T[x \ln (x)+(1-x) \ln (1-x)]
$$

is the related ideal Helmholtz free energy of mixing.

The nonideal behavior is solely based on HB redistribution, which is expressed by a partition function of the HB states for the cations, which are grouped into two distinct energy states with $\epsilon_{\mathrm{HB}, \mathrm{OMs}}=\Delta \epsilon_{\mathrm{HB}}$ and $\epsilon_{\mathrm{HB}, \mathrm{OTf}}=0$, populating the $c$ surrounding $\mathrm{HB}$ acceptence-sites according to the mixture composition with

$$
q_{\mathrm{HB}}^{N}=\left[c x^{\alpha} e^{-\beta \Delta \epsilon_{\mathrm{HB}}}+c\left(1-x^{\alpha}\right)\right]^{N},
$$

where $\beta=\left(k_{\mathrm{B}} T\right)^{-1}, N=N_{\text {[TEA] }}$, and $x=x_{\mathrm{OMs}}$. The parameter $\alpha$ is introduced to account for a potential nonstoichiometric availability of [OMs] and [OTf] HB acceptor sites within the mixture. Here $c x^{\alpha} e^{-\beta \Delta \epsilon_{\mathrm{HB}}}$ describe the HBs to the [OMs] anions and $c\left(1-x^{\alpha}\right)$ the corresponding HBs to the [OTf] anions. Hence the fraction of HB to the [OMs] anions is given by

$$
y=\frac{x^{\alpha} e^{-\beta \Delta \epsilon_{\mathrm{HB}}}}{x^{\alpha} e^{-\beta \Delta \epsilon_{\mathrm{HB}}}+1-x^{\alpha}}
$$

with $y=y_{\mathrm{OMs}}$, and $(1-y)=y_{\mathrm{OTf}}$. A statistical mechanical treatment of the partition function [35] given by Equation 9 is 


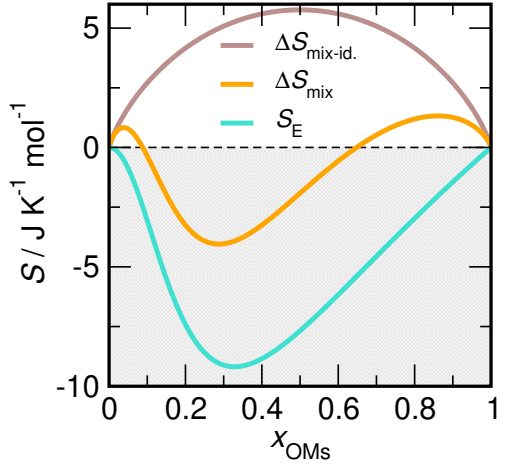

FIG. 5. Different contributions to the entropy of mixing $\Delta S_{\text {mix }}$ of the PIL mixture at $T=400 \mathrm{~K}$. The excess entropy $S_{\mathrm{E}}$ due to $\mathrm{HB}$ redistribution according to Equation 14 is negative, even overcompensating the positive ideal contribution $\Delta S_{\text {mix-id. }}$ due to the statistictical distribution of the anions in the mixture in the range $0.1 \leq x_{\mathrm{OMs}} \leq 0.65$.

leading directly to

$$
\begin{aligned}
U_{\mathrm{E}}= & N \Delta \epsilon_{\mathrm{HB}} \cdot \frac{x^{\alpha} e^{-\beta \Delta \epsilon_{\mathrm{HB}}}}{x^{\alpha} e^{-\beta \Delta \epsilon_{\mathrm{HB}}+1-x^{\alpha}}} \\
& -N x \Delta \epsilon_{\mathrm{HB}} \\
= & N \Delta \epsilon_{\mathrm{HB}} \cdot(y-x) .
\end{aligned}
$$

Note that Equation 12 is identical to Equation 5. The excess Helmholtz energy is accordingly

$$
\begin{aligned}
A_{\mathrm{E}}= & -x N \Delta \epsilon_{\mathrm{HB}} \\
& -N k_{\mathrm{B}} T \ln \left[x^{\alpha} e^{-\beta \Delta \epsilon_{\mathrm{HB}}}+1-x^{\alpha}\right] .
\end{aligned}
$$

From Equation 13 and Equation 11 follows the excess entropy as

$$
\begin{aligned}
S_{\mathrm{E}}= & N k_{\mathrm{B}} \ln \left[x^{\alpha} e^{-\beta \Delta \epsilon_{\mathrm{HB}}}+1-x^{\alpha}\right] \\
& +N k_{\mathrm{B}} y \frac{\Delta \epsilon_{\mathrm{HB}}}{k_{\mathrm{B}} T} .
\end{aligned}
$$

Figure 4 shows a comparison of the thermodynamic mixing properties obtained from MD simulations at $T=400 \mathrm{~K}$ with theoretical predictions employing $\Delta \epsilon_{\mathrm{HB}}=-10.5 \mathrm{~kJ} \mathrm{~mol}^{-1}$ and $\alpha=2.05$. We would like to point out that the behavior obtained at the highest temperature of $400 \mathrm{~K}$ is representative for the entire investigated temperature range, but is offering the best statistics. Obviously, the model is describing the simulation data very well using the energy difference $\Delta \epsilon_{\mathrm{HB}}$ obtained independently from the van 't Hoff analysis discussed in Figure 2. In addition, as shown in Figure 4b, the corresponding fractions of HBs to both anion species are described similarly well. This is strongly suggesting that $\mathrm{HB}$ redistribution is indeed the dominating contribution to the energy of mixing. The model, however, is also predicting the entropic contribution to the free energy of mixing. In particular, we would like to point out the curious negative mixing entropies $T \Delta S_{\text {mix }}$ observed in the range $0.1 \leq x_{\mathrm{OMs}} \leq 0.65$ in Figure 4a. As illustrated in Figure 5, this feature is observed because the excess entropy caused by HB redistribution effects according to Equation 14 is throughout negative and overcompensating the positive ideal contribution $\Delta S_{\text {mix-id. }}$ to the entropy of mixing. The fact that $S_{\mathrm{E}}<0$ can be understood as follows: the interaction $\Delta \epsilon_{\mathrm{HB}}$ is steering the HBs donated by the [TEA] cations towards [TEA]-[OMs] accepting sites and is therefore effectively reducing the orientational configuration space accessible to the cations in the mixture. This is contrasted by the situation in the pure liquid, where the HB-interactions with surrounding HB-acceptors all have the same energy. Here the cation can freely explore the HB-accepting sites of all the surrounding anions. By construction, the negative excess energy due to HB redistribution is connected with an excess entropy of the same sign, also called enthalpy-entropy compensation effect.[36] Such a behavior is often observed in phenomena, where hydrogen bonding is involved in equilibria affecting molecular (re-)organziation, such as hydrophobic hydration [37], or the thermodynamics of protein folding. [38]. A prominent example involving hydrogen bonding, where negative excess enthalpies and excess entropies correlate with one another, are of course aqueous alcohol mixtures.[39] In those systems, however, the excess entropies do not dominate the ideal mixing entropy. A solvent with the capability to donate and accept HBs and which is possessing the discussed thermodynamic features, however, could be of interest for a variety of applications in the field of "solvation science".[40] Finally, we would like to stress that the resulting Helmholtz energies of mixing of the PIL-systems are, of course, negative, leading to the formation of thermodynamically stable mixtures. In addition to the purely theoretical considerations outlined above, we will demonstrate in a forthcoming paper that both, experimental and simulated mixtures behave qualitatively similar with respect to the enthalpic and structural effects associated with HB redistribution.[41] In conclusion, this particular mixture of PILs seems to be one of few examples with a negative mixing entropy [42] and where where the mixing process is driven by enthalpy, not entropy.

We thank the Leibniz Association, the State of Mecklenburg-Vorpommern, and the University of Rostock for financial support within the ComBioCat programme. This work has been supported by a DFG Research Grant (No. 401427621). D.O. acknowledges the support from the grant No. A1_FCHI_2021_002 of specific university research by the Ministry of Education, Youth and Sports (Czech Republic).

* dietmar.paschek@uni-rostock.de

[1] J.-P. Belieres and C. A. Angell. Protic ionic liquids: Preparation, characterization, and proton free energy level representation. J. Phys. Chem. B, 111:4926-4937, 2007.

[2] R. Caparica, A. Júlio, A. R. Baby, E. U. A. M. Araújo, A. S. Fernandes, J. G. Costa, and T. Santos de Almeida. Choline-amino acid ionic liquids as green functional excipients to enhance drug solubility. Pharmaceutics, 10:288, 2018.

[3] B. Peric, S. Sierra, E. Martí, R. Cruanas, M. A. Garau, 
J. Arning, U. Bottin-Weber, and S. Stolte. (eco)toxicity and biodegradability of selected protic and aprotic ionic liquids. $J$. Hazard Mater., 261:99-105, 2013.

[4] H. Every, A. G. Bishop, M. Forsyth, and D. R. MacFarlane. Ion diffusion in molten salt mixtures. Electrochimica Acta, 45:1279-1284, 2000.

[5] K. A. Fletcher, S. N. Baker, G. A. Baker, and S. Pandey. Probing solute and solvent interactions within binary ionic liquid mixtures. New J. Chem., 27:1706-1712, 2003.

[6] J. N. Canongia Lopes, T. C. Cordeiro, J. M. S. S. Esperanca, H. J. R. Guedes, S. Huq, L. P. N. Rebelo, and K. R. Seddon. Deviations from ideality in mixtures of two ionic liquids containing a common ion. J. Phys. Chem. B, 109:3519-3525, 2005.

[7] P. Navia, J. Troncoso, and L. Romaní. Viscosities fir ionic liquid binary mixtures with a common ion. J. Solution Chem., 37:677688, 2008

[8] Gary Annat, M. Forsyth, and D. R. MacFarlane. Ionic liquid mixtures - variations in physical properties and their origins in molecular structure. J. Phys. Chem. B., 116:8251-8258, 2012.

[9] M. B. Oliveira, M. Domínguez-Pérez, O. Cabeza, J. A. Lopes da Silva, M. G. Freire, and J. A. P. Coutinho. Surface tensions of binary mixtures of ionic liquids with bis(trifluoromethylsulfonyl)imide as the common anion. $J$. Chem. Thermodynamics, 64:22-27, 2013.

[10] R. S. Payal and S. Balasubramanian. Homogenous mixing of ionix liquids: Molecular dynamics simulations. Phys. Chem. Chem. Phys., 15:21077-21083, 2013.

[11] D. Paschek, B. Golub, and R. Ludwig. Hydrogen bonding in a mixture of protic ionic liquids: A molecular dynamics simulation study. Phys. Chem. Chem. Phys., 17:8431-8440, 2015.

[12] K. Fumino, A.-M. Bonsa, B. Golub, D. Paschek, and R. Ludwig. Non-ideal mixing behaviour of hydrogen bonding in mixtures of protic ionic liquids. ChemPhysChem, 16:299-304, 2015.

[13] R. P. Matthews, I. J. Villar-Garcia, C. C. Weber, J. Griffith, F. Cameron, J. P. Hallett, P. A. Hunt, and T. Welton. A structural investigation of ionic liquid mixtures. Phys. Chem. Chem. Phys., 18:8608-8624, 2016

[14] D. W. Bruce, C. P. Cabry, J. N. Canongia Lopes, M. L. Costen, L. D'Andrea, I. Grillo, B. C. Marshall, K. G. McKendrick, T. K. Minton, S. M. Purcell, S. Rogers, J. M. Slattery, K. Shimizu, E. Smoll, and M. A. Tesa-Serrate. Nanosegregation and strcturing in the bulk and at the surface of ionic-liquid mixtures. $J$. Phys. Chem. B, 121:6002-6020, 2017.

[15] T. Vogl, S. Passerini, and A. Balducci. The impact of mixtures of protic ionic liquids on the operative temerature range of use of battery systems. Electrochem commun, 78:47-50, 2017.

[16] C. P. Cabry, L. D’Andrea, K. Shimizu, I. Grillo, P. Li, S. Rogers, D. W. Bruce, J. N. Canongia Lopes, and J. M. Slattery. Exploring the bulk-phase structure of ionic liquid mixtures using small-angle neutron scattering. Faraday Discuss., 206:265289, 2018.

[17] S. Thawarkar, N. D. Khupse, and A. Kumar. Binary mixtures of aprotic and protic ionic liquids demonstrate synergistic polarity effect: An unusual observation. J. Sol. Chem., 49:210-221, 2020.

[18] E. P. Yambou, B. Gorska, and F. Béguin. Binary mixtures of ionic liquids based on emim cation and fluorinated anions: Physico-chemical characterization in view of their application as low-temperature electrolytes. J. Mol. Liq., 298:111959-, 2020.

[19] M. Chakraborty, S. Barik, A. Mahapatra, and M. Sarkar. Binary mixtures of ionic liquids: Ideal, non-ideal, or quasi-ideal? $J$. Chem. Phys., 154:224507, 2021.
[20] H. Niedermeyer, J. P. Hallett, I. J. Villar-Garcia, P. A. Hunt, and T. Welton. Mixtures of ionic liquids. Chem. Soc. Rev., 41:77807802, 2012.

[21] A. Podgorsek, A. S. Pensado, C. C. Santini, M. F. Costa Gomes, and A. A. H. Pádua. Interaction energies of ionic liquids with metallic nanoparticles: Solvation and stabilization effects. $J$. Phys. Chem. C, 117:3537-3547, 2013.

[22] L. F. Lepre, J. Szala-Bilnik, A. A. H. Padua, M. Traikia, R. A. Ando, and M. F. Costa Gomes. Tailoring the properties of acetate-based ionic liquids using the tricyanomethanide anion. Phys. Chem. Chem. Phys., 18:23285-23295, 2016.

[23] C. Herrera, M. Atilhan, and S. Aparicio. A theoretical study on mixtures of amino acid-based ionic liquids. Phys. Chem. Chem. Phys., 20:10213-10223, 2018.

[24] P. Navia, J. Troncoso, and L. Romaní. Excess magnitudes for ionic liquid binary mixtures with a common ion. J. Chem. Eng. Data, 52:1369-1374, 2007.

[25] H. J. Castejón and R. J. Lashock. Mixtures of ionic liquids with similar molar volumes form regular solutions and obey the cross-square rules for eectrolyte mixtures. J. Mol. Liq., 167:14, 2012.

[26] A.Podgorsek, J. Jacquemin, A. A. H. Pádua, and M. F. Costa Gomes. Mixing enthalpy for binary mixtures containing ionic liquids. Chem. Rev., 116:6075-6106, 2016.

[27] I. Otero, L. F. Lepre, A. Dequidt, P. Husson, and M. F. Costa Gomes. How does the addition of a third ion affect the molecular interations and the thermodynamic properties to acetatebased ionic liquids? J. Phys. Chem. B, 121:9725-9736, 2017.

[28] B. Docampo-Álvarez, V. Gómez-González, T. MéndezMorales, J. R. Rodríguez, , O. Cabeza, M. Turmine, L. J. Gallego, and L. M. Varela. The effect of alkyl chain length on the structure and thermodynamics of protic-aprotic ionic liquid mixtures: A molecular dynamics study. Phys. Chem. Chem. Phys., 20:9938-9949, 2018.

[29] L. F. Lepre, M. F. Costa Gomes, A. A. H. Pádua, R. A. Ando, and M. C. C. Ribeiro. On the regular behavior of a binary mixture of ionic liquids. J. Phys. Chem. B, 123:6579-6587, 2019.

[30] G. Chatel, F. F. B. Pereira, V. Debbeti, H. Wang, and R. D. Rogers. Mixing ionic liquids - simple mixture or double salts? Green Chem., 16:2051-2083, 2014.

[31] R. Caminiti and L. Gontrani, editors. The Structure of Ionic Liquids. Springer, 2014.

[32] B. Golub, K. Fumino, P. Stange, V. Fossog, R. Hempelmann, D. Ondo, D. Paschek, and R. Ludwig. Balance between contact and solvent separated ion pairs in mixtures of the protic ionic liquid $\left[\mathrm{Et}_{3} \mathrm{NH}\right]\left[\mathrm{MeSO}_{3}\right]$ with water controlled by water content and temperature. J. Phys. Chem. B, 125:4476-4488, 2021.

[33] K. Fumino, V. Fossog, K. Wittler, R. Hempelmann, and R. Ludwig. Dissecting anion-cation interation energies in protic ionic liquids. Angew. Chem. Int. Ed., 52:2368-2372, 2013.

[34] E. Bodo, M. Bonomo, and A. Mariani. Assessing the structure of protic ionic liquids based on triethylammonium and organic acid anions. J. Phys. Chem. B, 125:2781-2792, 2021.

[35] T. L. Hill. An Introduction to Statistical Thermodynamics. Dover, 1960.

[36] H. Qian and J. J. Hopfield. Entropy-enthalpy compensation: Perturbation and relaxation in thermodynamic systems. $J$. Chem. Phys., 105:9292-9298, 1996.

[37] K. Koga, B. Bhimalapuram, and B. Widom. The hydrophobic effect. Phys. Chem. Chem. Phys., 5:3085-3093, 2003.

[38] K. A. Dill and J. A. MacCallum. The protein-folding problem, 50 years on. Science, 338:1042-1046, 2012.

[39] R. F. Lama and L. Benjamin. Excess thermodynamics properties of aqueous alcohol solutions. J. Chem. Eng. Data, 10:216- 
219,1965

[40] M. Havenith. Solvation science: A new interdisciplinary field. Angew. Chem. Int. Ed., 55:1218-1219, 2016.

[41] B. Golub, D. Ondo, V. Overbeck, R. Ludwig, and D. Paschek. Hydrogen bond redistribution effects in mixtures of protic ionic liquids sharing the same cation: Nonideal mixing with large negative mixing enthalpies. Preprint, 2022.

[42] O. Delaire, T. S. Swan-Wood, and B. Fultz. Negative entropy of mixing for vanadium-platinum solutions. Phys. Rev. Lett., 93:185704, 2004. 